\title{
Global CALIPSO Observations of Aerosol Changes Near Clouds
}

\author{
TamásVárnai and Alexander Marshak
}

\begin{abstract}
Several recent studies have found that clouds are surrounded by a transition zone of rapidly changing aerosol optical properties and particle size. Characterizing this transition zone is important for better understanding aerosol-cloud interactions and aerosol radiative effects, and also for improving satellite retrievals of aerosol properties. This letter presents a statistical analysis of a monthlong global data set of Cloud-Aerosol Lidar and Infrared Pathfinder Satellite Observation (CALIPSO) lidar observations over oceans. The results show that the transition zone is ubiquitous over all oceans and extends up to $15 \mathrm{~km}$ away from clouds. They also show that near-cloud enhancements in backscatter and particle size are strongest at low altitudes, slightly below the top of the nearest clouds. Also, the enhancements are similar near illuminated and shadowy cloud sides, which confirms that the asymmetry of Moderate Resolution Imaging Spectroradiometer refiectances found in an earlier study comes from 3-D radiative processes and not from differences in aerosol properties. Finally, the effects of CALIPSO aerosol detection and cloud identification uncertainties are discussed. The findings underline the importance of accounting for the transition zone to avoid potential biases in studies of satellite aerosol products, aerosol-cloud interactions, and aerosol direct radiative effects.
\end{abstract}

Index Terms-Aerosol, cloud, Cloud-Aerosol Lidar and Infrared Pathfinder Satellite Observation (CALIPSO), lidar, satellite.

\section{INTRODUCTION}

$\mathbf{S}$ EVERAL recent studies have indicated that clouds are surrounded by a transition zone of rapidly changing aerosol optical properties and particle size (e.g., [1]-[5]). Characterizing this transition zone is important for better understanding two critical yet poorly understood aspects of anthropogenic climate change-aerosol-cloud interactions and aerosol radiative effects-[6], and also for devising effective sampling strategies for satellite measurements of aerosol properties.

Satellites offer excellent opportunities to study the transition zone near clouds, although the separation between clouds and cloud-free areas is often uncertain, and ambiguities can occur at all scales [7]. As Charlson et al. [8] summed up, "Multiple

Manuscript received January 20, 2010; revised March 8, 2010; accepted March 29, 2010. Date of publication June 21, 2010. This work was supported by the National Aeronautics and Space Administration (NASA) Radiation Sciences Program managed by Hal Maring.

T. Varnai is with the Joint Center for Earth Systems Technology, University of Maryland, Baltimore, MD 21228 USA, and also with the NASA Goddard Space Flight Center, Greenbelt, MD 20771 USA (e-mail: tamas.varnai@nasa.gov).

A. Marshak is with the NASA Goddard Space Flight Center, Greenbelt, MD 20771 USA (e-mail: Alexander.marshak @nasa.gov).

Color versions of one or more of the figures in this paper are available online at http://ieeexplore.ieee.org.

Digital Object Identifier 10.1109/LGRS.2010.2049982 lines of evidence exist that call into question the degree to which clear and cloudy sky can be separated...". Consequently, improving our knowledge of the transition zone requires improvements in understanding and separating remote sensing uncertainties and physical phenomena such as aerosol swelling in humid air.

One reason why such improvements are important is that aerosol changes and remote sensing uncertainties near clouds create a dilemma: Excluding the transition zone in order to avoid its remote sensing uncertainties can bias a study toward low aerosol optical depths, thus weaker radiative effects calculated from these optical depths, but including the transition zone despite the remote sensing uncertainties can bias the study toward too high aerosol optical depths and stronger radiative effects.

Lidar instruments offer excellent opportunities for studying the transition zone, because their measurements are not affected by 3-D cloud radiative effects or instrument blurring (e.g., [9]-[12]). Recently, Tackett and Di Girolamo [13] pioneered the use of the spaceborne Cloud-Aerosol Lidar and Infrared Pathfinder Satellite Observation (CALIPSO) lidar for studying the transition zone. Their study of aerosols over the Caribbean Sea showed that aerosol properties change rapidly near clouds both because aerosols swell in the humid environment and because the collision and coalescence of aerosol-containing cloud droplets and their subsequent evaporation produce fewer but larger aerosol particles. Additionally, aerosol nucleation by aqueous-phase reactions is also a factor.

While the pioneering study of Tackett and Di Girolamo [13] focused on areas up to 3-4 km away from clouds in the Caribbean, this letter analyzes a large global data set of CALIPSO measurements, which can reveal even the subtle changes that occur at the more distant portions of the transition zone.

\section{DATA AND METhodology}

This letter analyzes a monthlong (from September 15 to October 14, 2008) global data set of CALIPSO lidar data over all oceans free of sea ice. Our statistical analysis uses the operational 1-km-resolution CALIPSO cloud mask and 333-mresolution attenuated backscatter profiles at both 532- and 1064-nm wavelengths. Most results shown are for nighttime data simply because the weaker background illumination creates smaller noise and allows more reliable cloud detection, but, as it will be illustrated in the last figure, daytime results showed very similar behaviors.

Following Tackett and Di Girolamo [13], we characterize the transition zone by examining how median attenuated backscatter values vary with distance from the nearest cloud. Median values are well suited for this purpose because they are less 

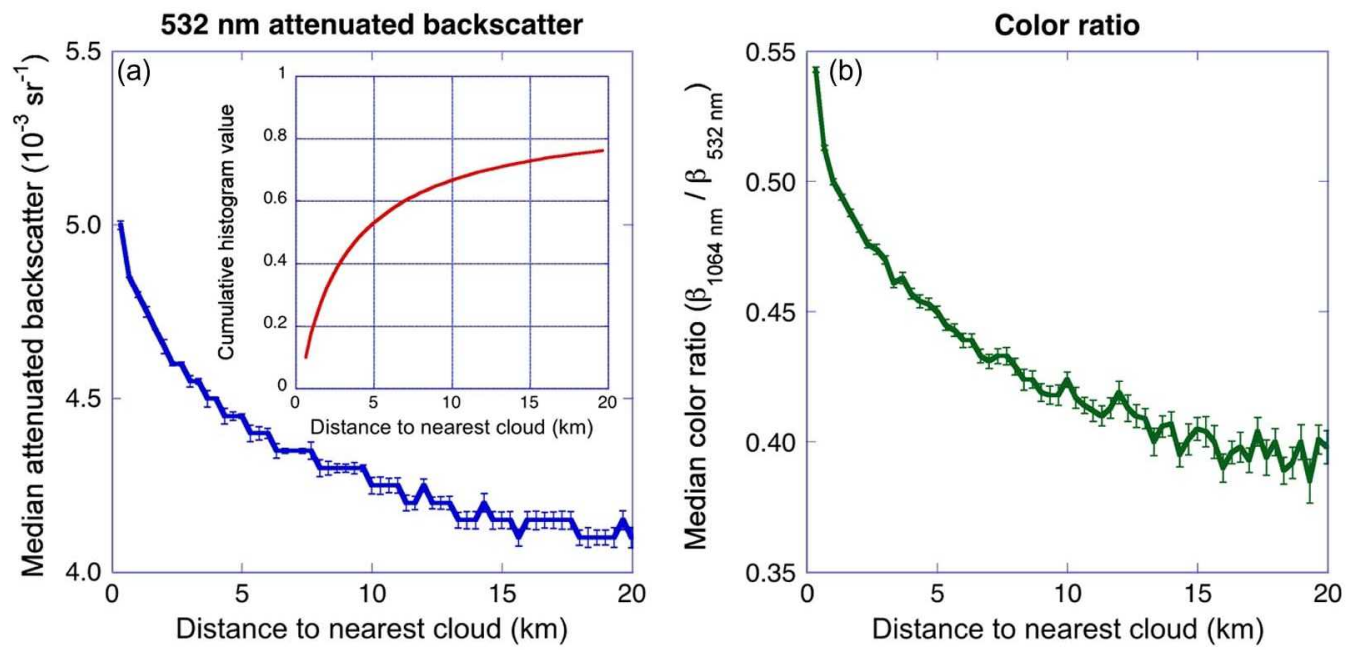

Fig. 1. (a) Global median vertically integrated attenuated backscatter for all nighttime CALIPSO observations over ice-free oceans. (Inset) Cumulative histogram of cloud-free profiles. (b) Median color ratio. These and subsequent plots are based on a monthlong data set in September-October 2008 . The distances in all figures are to the nearest cloud below 3-km altitude. The error bars in this and subsequent figures indicate uncertainties estimated using the bootstrap method [19]. Because this method assumes that all profiles are independent samples, it represents a lower limit to actual uncertainties.

affected than mean values by uncertainties in cloud detection and by unobserved clouds that lie right near the satellite track. We calculate median values by identifying the 50th-percentile points in high-resolution backscatter and color ratio histograms. Let us note that, although this letter shows only median values, mean values show generally similar but noisier behaviors.

This letter focuses on clear areas closest to low-level clouds (below $3 \mathrm{~km}$ ), but we note that results for clear areas near any clouds were very similar to those presented here. One reason for the similarity appears in the inset of Fig. 1(a), which shows the cumulative histogram of clear-sky profiles as a function of distance to the nearest cloud that has a cloud top below $3 \mathrm{~km}$. The figure shows that $50 \%$ of all clear profiles are closer than $4.4 \mathrm{~km}$ to a cloud below $3 \mathrm{~km}$, and $75 \%$ of them are closer than $20 \mathrm{~km}$. The remaining $25 \%$ of clear profiles include both those clear profiles that are closest to a cloud higher than $3 \mathrm{~km}$ and those clear profiles that are more than $20 \mathrm{~km}$ away from any cloud. We note that Twohy et al. [5] found $66 \%$ of all clear areas they observed over the Indian Ocean to be closer than $4 \mathrm{~km}$ to clouds.

Our results indicate that much more clear profiles lie close to clouds below $3 \mathrm{~km}$ than to higher clouds, even though the highest cloud top is more often above than below $3 \mathrm{~km}$ ( $40 \%$ versus $38 \%$ of all CALIPSO profiles in our data set). This occurs because high clouds often form large continuous layers, and so, only a few clear profiles can be close to high clouds (at the two ends of the large cloud decks) - whereas low cloud layers often consist of numerous small clouds separated by clear gaps, which allows many clear profiles to be close to them. The CALIPSO cloud detection algorithm [14] may further emphasize this tendency when it reduces the effects of observational noise by detecting faint (and often high) clouds after averaging observed backscatter values over larger scales, which fills in any small gaps in thin high clouds.

Finally, we note that we show results of attenuated backscatter values integrated up to $3-\mathrm{km}$ altitude and that the results behaved very similarly when integrating to $15-\mathrm{km}$ altitude. We chose to present the results for low-level backscatter because this is where the bulk of near-cloud aerosol changes occur.

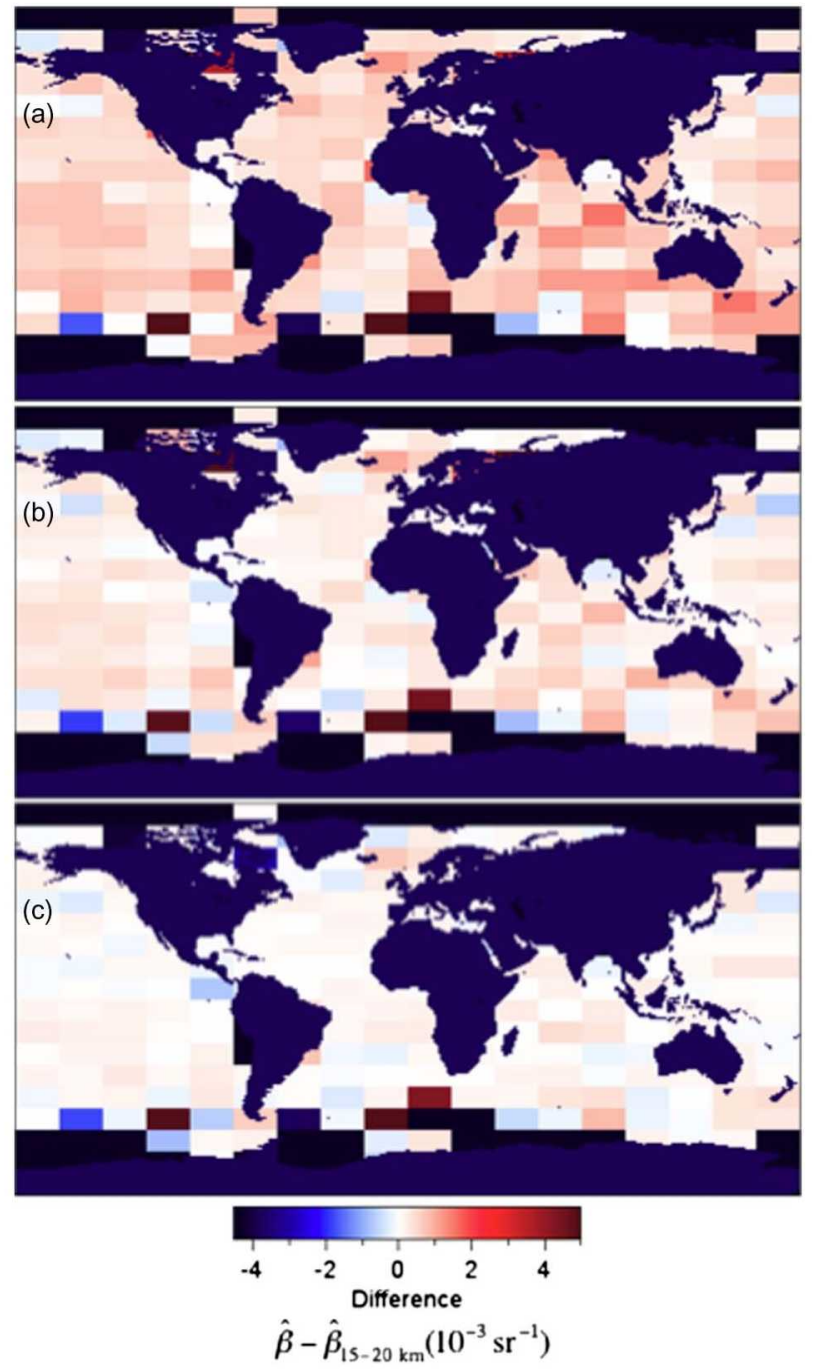

Fig. 2. Map of differences between median vertically integrated $532-\mathrm{nm}$ backscatter values $(\hat{\beta})$ at different distances from the nearest cloud. (a) $\hat{\beta}_{0-5 \mathrm{~km}}-\hat{\beta}_{15-20 \mathrm{~km}}$. (b) $\hat{\beta}_{5-10 \mathrm{~km}}-\hat{\beta}_{15-20 \mathrm{~km}}$. (c) $\hat{\beta}_{10-15 \mathrm{~km}}-$ $\hat{\beta}_{15-20 \mathrm{~km}}$. 

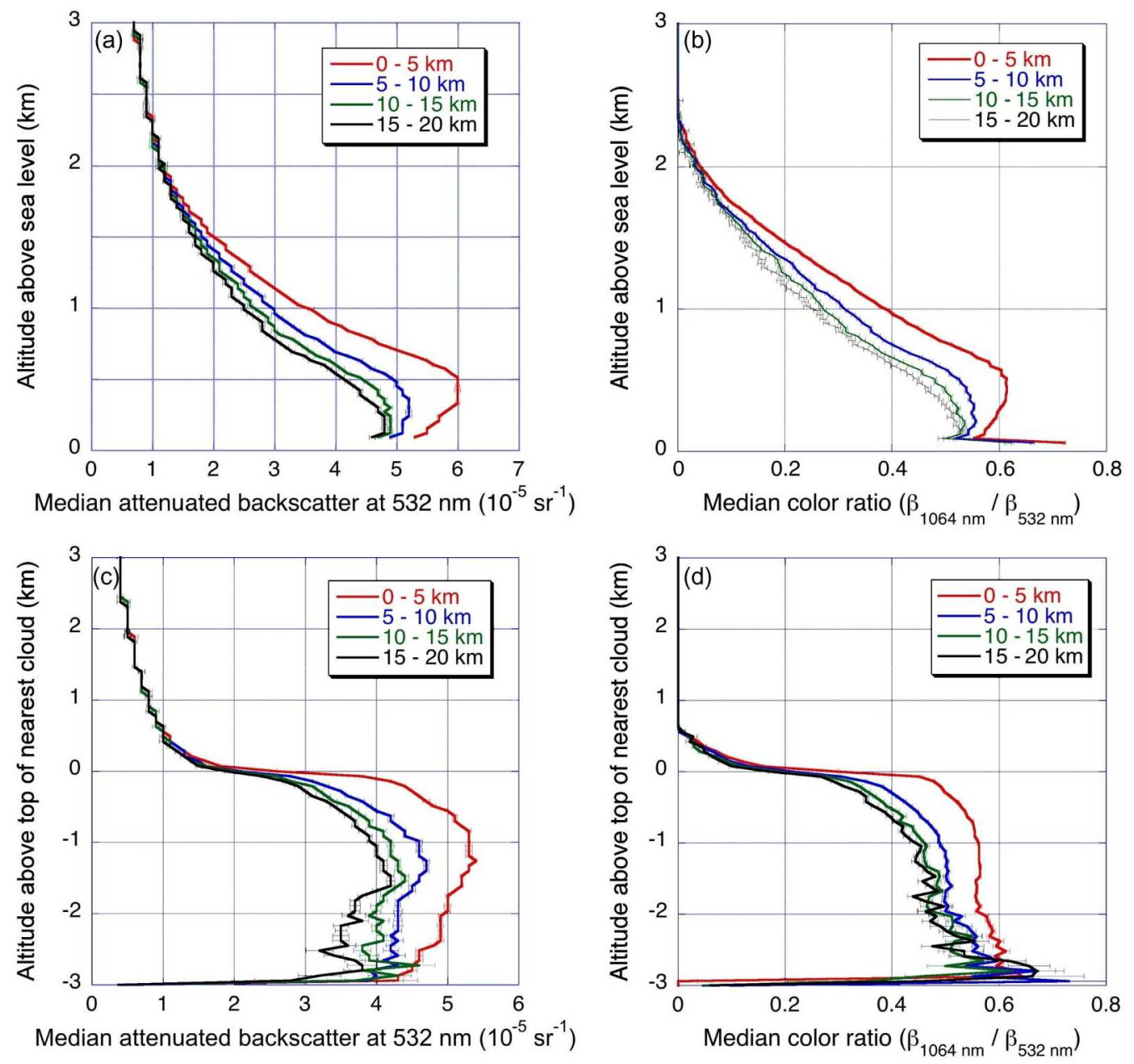

Fig. 3. Altitude dependence of median enhancements near clouds. (a) 532-nm attenuated backscatter versus altitude above sea level. (b) Color ratio versus altitude above sea level. (c) 532-nm attenuated backscatter versus altitude above the top of the nearest cloud. (d) Color ratio versus altitude above the top of the nearest cloud.

\section{Results on Particles Near Clouds}

Fig. 1(a) confirms the overall tendency found in earlier papers that attenuated backscatter increases systematically toward clouds. The figure indicates that this tendency is sufficiently ubiquitous to appear in global statistics and that the transition zone typically extends to about $15 \mathrm{~km}$.

Fig. 1(b) shows that the spectral behavior of attenuated backscatter $(\beta)$ also changes near clouds: The median "color ratio" (which is slightly smaller than the ratio of medians at the two wavelengths but follows the same behavior) increases systematically near clouds. Because backscatter decreases with wavelength faster for smaller particles than for larger particles (i.e., smaller particles have larger Angstrom exponents), the color ratio increase in Fig. 1(b) indicates that particle size increases near clouds. This increase is consistent with the effects of both aerosol humidification and cloud detrainment, but establishing the relative importance of these two processes as in [13] will require a more detailed study.

Fig. 2 shows that the increase near clouds in Fig. 1 is a global phenomenon and that the overall trend is neither caused by a single dominant region nor is it an artifact of combining observations from vastly different regions of the Earth. While undoubtedly there are regional variations, much of the variations in Fig. 2 likely come from sampling issues.
Fig. 3 shows the vertical structure of near-cloud backscatter enhancements by comparing the altitude dependence of backscatter at four intervals of distance to clouds $(0-5,5-10$, $10-15$, and 15-20 km). Panels (a) and (b) of the figure show that aerosol content and size (i.e., 532-nm backscatter and color ratio) both decrease with altitude and that the near-cloud enhancements also decrease with altitude. The exception is a small increase at very low altitudes, a trend most pronounced at tropical areas.

Panels (c) and (d) of the figure show the way backscatter and color ratio depend on altitude relative to the top of the nearest cloud. They reveal that aerosol content and size drop sharply above cloud altitude, as convection is not as effective in moving aerosols from low levels to above cloud tops. Nearcloud enhancements are also much larger below than above the cloud top. The maximum enhancement at cloud altitude is consistent with cloud detrainment occurring here by default, with aerosol humidification being strongest when humidity approaches saturation (e.g., [5]).

\section{EFFeCts of Remote Sensing Limitations NeAR CloudS}

While aerosol particles are responsible for a large part of the near-cloud enhancements discussed earlier, misidentified or 

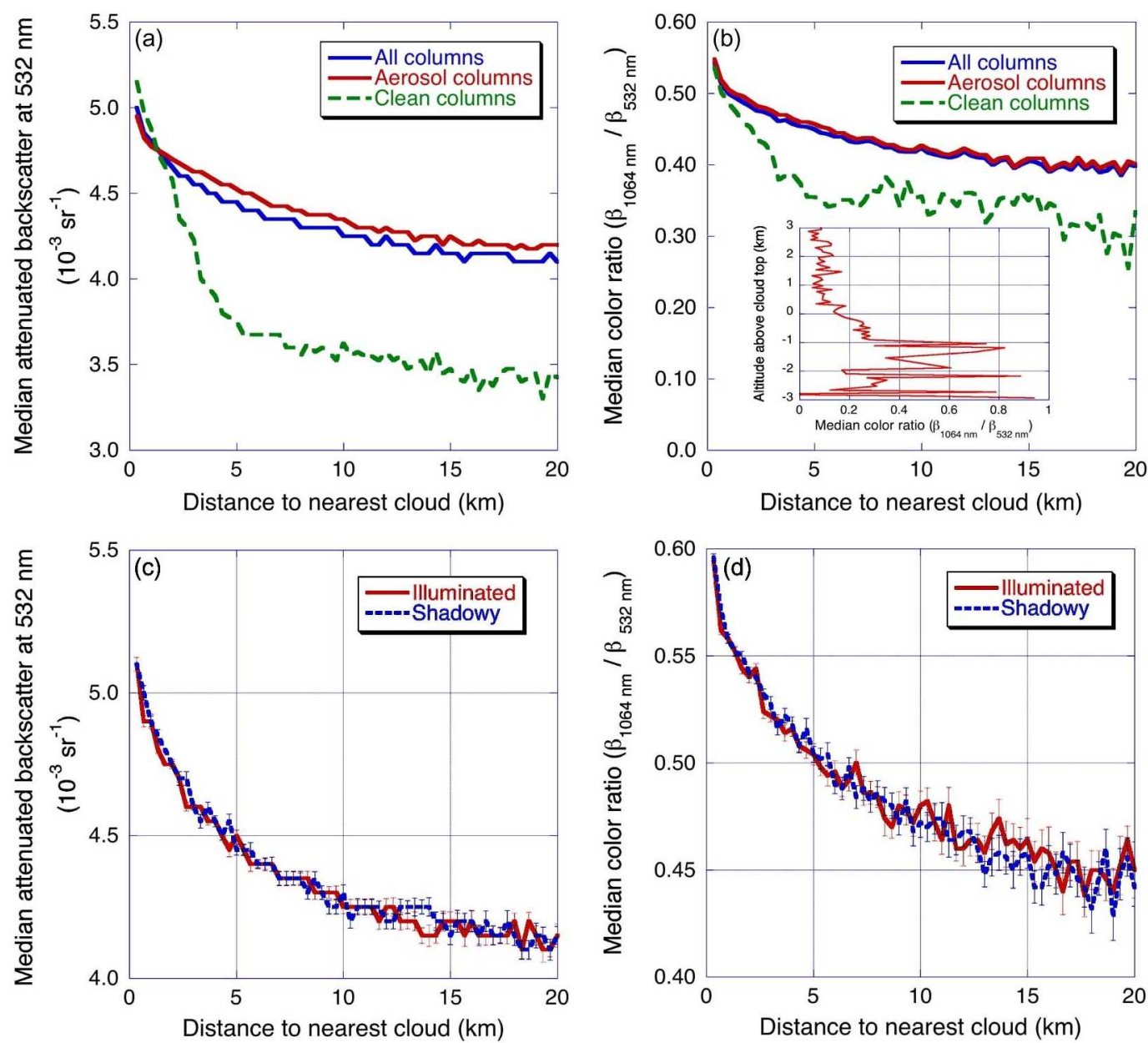

Fig. 4. Results on remote sensing uncertainties near clouds. (a) and (b) Median vertically integrated 532-nm attenuated backscatter and color ratio for nighttime CALIPSO observations over ice-free oceans. These panels consider separately the atmospheric columns that are free of both cloud and aerosol layers and the columns that contain an aerosol layer, as specified by the operational CALIPSO product. The inset in (b) shows the vertical profile of median color ratio in atmospheric columns that are 15-20 km away from the nearest detected clouds and where the operational CALIPSO product did not detect any aerosol layers. (c) and (d) Median near-cloud vertically integrated 532-nm attenuated backscatter and color ratio in daytime observations over all oceans between $30^{\circ}$ and $60^{\circ}$ latitudes in both hemispheres. The solid and dashed lines are for profiles that are on the sunlit and shadowy sides of the closest cloud, respectively.

undetected cloud particles are also likely to contribute. Undetected cloud particles contribute to clear-sky statistics because the separation of cloudy and cloud-free areas is sometimes ambiguous due to uncertainties both in detecting layers of scattering particles and in distinguishing clouds from aerosols [14]. Identifying cloud particles is particularly difficult when small clouds cover only a portion of the lidar field of view-a situation that often occurs in sparse cumulus fields, where Koren et al. [7] found very small clouds to be fairly abundant. Although using medians instead of mean values greatly reduces the influence of cloud detection errors, we note that an ideal cloud mask would slightly weaken the near-cloud enhancements. Another aspect of cloud detection uncertainties is that clouds that lie next to the lidar path do not get detected, which causes an overestimation of the distance to the nearest cloud. This results in somewhat overestimating the width of the transition zone.

As an example of remote sensing issues, Fig. 4(a) and (b) shows that lidar backscatter and color ratio increase near clouds even in pristine atmospheric columns where the operational CALIPSO product did not detect any aerosol layers. In comparison to all cloud-free profiles, the increase in pristine areas is particularly well pronounced within $4-5 \mathrm{~km}$ from the clouds identified by CALIPSO. An unexpected feature of Fig. 4(a) is that backscatter right next to clouds is higher for pristine areas than for areas with detected aerosol layers. A possible reason why aerosol is not detected despite the higher backscatter values is that, in the coarse-resolution ( $\geq 5-\mathrm{km})$ CALIPSO aerosol detections, lower "pristine area" backscatter farther away from clouds pushes area-averaged backscatter values below the aerosol detection threshold. The inset in Fig. 4(b) shows that color ratios below the cloud top greatly exceed the value of $\sim 0.07$ that is expected from clear air molecular scattering (e.g., [14]). These results indicate that aerosol or cloud particles are often present even in these pristine profiles.

Therefore, while particle backscatter and size certainly increase near the clouds detected by CALIPSO, both the nature of these particles (cloud versus aerosol) and the processes creating them (e.g., aerosol swelling in humid air) need to be clarified using a quantitative assessment of remote sensing limitations in particle detection and classification.

Finally, Fig. 4(c) and (d) confirms the importance of remote sensing effects that influence satellite measurements of solar reflectance near clouds. In particular, the figures show daytime 
near-cloud enhancements of lidar backscatter between $30^{\circ}$ and $60^{\circ}$ latitudes. Each panel contains two curves: one for clearsky profiles that are on the sunlit side of the nearest cloud (toward the Equator from the cloud) and the other that are on the shadowy side of the nearest cloud (toward the poles from the cloud). The figure indicates that the increase is very similar on both sides of clouds. This confirms that the increase being larger near sunlit cloud sides in Moderate Resolution Imaging Spectroradiometer (MODIS) solar reflectance observations is most likely a result of 3-D radiative processes [12], as opposed to asymmetric changes in aerosol properties. This implies that 3-D radiative effects are important in shaping clear-sky radiation fields near clouds and should be accounted for in interpreting passive space-based aerosol measurements.

\section{Conclusive Thoughts}

This letter presents a statistical analysis of a monthlong global data set of CALIPSO lidar observations over oceans. The results show the following.

- The transition zone of enhanced backscatter and particle size around clouds is ubiquitous over all oceans.

- The transition zone is much wider $(\sim 15 \mathrm{~km})$ than the ranges examined in most earlier studies.

- Near-cloud enhancement is strongest at low altitudes, slightly below the top of the nearest clouds.

In addition, the results also provide information on uncertainties in aerosol remote sensing near clouds.

- CALIPSO enhancements are similar near illuminated and shadowy cloud sides, confirming that the differences found in an earlier study of MODIS solar reflectances [12] indeed come from 3-D radiative effects and not from differences in aerosol properties.

- Aerosol or cloud particles cause backscatter and color ratio enhancements near clouds even in pristine profiles where the CALIPSO products did not detect any aerosol or cloud.

The width and ubiquity of the transition zone near clouds imply that studies of aerosol-cloud interactions and aerosol direct radiative effects need to quantitatively account for aerosol changes near clouds. These changes can cause systematic biases toward smaller aerosol optical depths, thus weaker radiative forcings calculated from these optical depths, if areas near clouds are not included in order to avoid aerosol retrieval ambiguities that arise near clouds (e.g., 3-D radiative effects, instrument blurring, and cloud contamination). On the other hand, including aerosol products near clouds despite these ambiguities overestimates aerosol radiative forcing. As a result, there is an urgent need for developing methods that can assess and account for remote sensing challenges (e.g., for passive remote sensing, [15], [16]) and thus allow for including the transition zone into the study. Alternatively, one may remove biases by seeking a balanced sampling strategy that may compensate for excluding both near-cloud and far-from-cloud areas to a similar degree (e.g., [17]). Finally, statistical adjustments (e.g., [18]) of results limited to areas far from clouds may also be possible if the statistical behavior of the transition zones becomes well understood. More detailed future analysis of transition zones is expected to provide further insights into their origins and influence on the atmosphere, radiative processes, and satellite data products.

\section{ACKNOWLEDGMENT}

The authors would like to thank C. Chiu, J. A. Coakley, A. B. Davis, L. Di Girolamo, R. E. Holz, G. Wen, and W. Yang for the highly productive and stimulating discussions.

\section{REFERENCES}

[1] I. Koren, L. A. Remer, Y. J. Kaufman, Y. Rudich, and J. V. Martins, "On the twilight zone between clouds and aerosols," Geophys. Res. Lett., vol. 34, p. L08 805, Apr. 2007, DOI: 10.1029/2007GL029253.

[2] W. Su, G. L. Schuster, N. G. Loeb, R. R. Rogers, R. A. Ferrare, C. A. Hostetler, J. W. Hair, and M. D. Obland, "Aerosol and cloud interaction observed from high spectral resolution lidar data," J. Geophys. Res., vol. 113, p. D24 202, Dec. 2008, DOI: 10.1029/2008JD010588.

[3] J. C. Chiu, A. Marshak, Y. Knyazikhin, P. Pilewskie, and W. J. Wiscombe, "Physical interpretation of the spectral radiative signatures in the transition zone between cloud-free and cloudy regions," Atmos. Chem. Phys., vol. 9, no. 5, pp. 1419-1430, Sep. 2008.

[4] J. Redemann, Q. Zhang, P. B. Russell, J. M. Livingston, and L. A. Remer, "Case studies of aerosol remote sensing in the vicinity of clouds," J. Geophys. Res., vol. 114, no. D6, p. D06209, Mar. 2009, DOI: $10.1029 / 2008$ JD010774.

[5] C. H. Twohy, J. A. Coakley, Jr., and W. R. Tahnk, "Effect of changes in relative humidity on aerosol scattering near clouds," J. Geophys. Res., vol. 114, p. D05 205, Mar. 2009, DOI:10.1029/2008JD010991.

[6] N. G. Loeb and G. L. Schuster, "An observational study of the relationship between cloud, aerosol and meteorology in broken low-level cloud conditions," J. Geophys. Res., vol. 113, p. D14214, Jul. 2008, DOI: 10.1029/2007JD009763.

[7] I. Koren, L. Oreopoulos, G. Feingold, L. A. Remer, and O. Altaratz, "How small is a small cloud?," Atmos. Chem. Phys., vol. 8, pp. 3855-3864, 2008.

[8] R. J. Charlson, A. S. Ackerman, F. A.-M. Bender, T. L. Anderson, and Z. Liu, "On the climate forcing consequences of the albedo continuum between cloudy and clear air," Tellus, vol. 59, no. 7, pp. 715-727, Sep. 2007.

[9] S. Qiu, G. Godden, X. Wang, and B. Guenther, "Satellite-Earth remote sensor scatter effects on Earth scene radiometric accuracy," Metrologia, vol. 37, no. 5, pp. 411-414, Nov. 2000.

[10] J. Zhang, J. S. Reid, and B. N. Holben, "An analysis of potential cloud artifacts in MODIS over ocean aerosol optical thickness products," Geophys. Res. Lett., vol. 32, p. L15 803, Aug. 2005, DOI: 10.1029/2005GL023254.

[11] G. Wen, A. Marshak, R. F. Cahalan, L. A. Remer, and R. G. Kleidman, "3-D aerosol-cloud radiative interaction observed in collocated MODIS and ASTER images of cumulus cloud fields," J. Geophys. Res., vol. 112, no. D13, p. D13 204, Jul. 2007, DOI: 10.1029/2006JD008267.

[12] T. Várnai and A. Marshak, "MODIS observations of enhanced clear sky reflectance near clouds," Geophys. Res. Lett., vol. 36, p. L06807, Mar. 2009, DOI: 10.1029/2008GL037089.

[13] J. L. Tackett and L. Di Girolamo, "Enhanced aerosol backscatter adjacent to tropical trade wind clouds revealed by satellite-based lidar," Geophys. Res. Lett., vol. 36, no. 14, p. L14 804, Jul. 2009, DOI: 10.1029/2009GL039264.

[14] Z. Liu, M. A. Vaughan, D. M. Winker, C. A. Hostetler, L. R. Poole, D. Hlavka, W. Hart, and M. McGill, "Use of probability distributions for discriminating between cloud and aerosol in lidar backscatter data," J. Geophys. Res., vol. 109, p. D15 202, 2004, DOI: 10.1029/ 2004JD004732.

[15] E. I. Kassianov and M. Ovtchinnikov, "On reflectance ratios and aerosol optical depth retrieval in the presence of cumulus clouds," Geophys. Res. Lett., vol. 35, p. L06 311, Mar. 2008, DOI: 10.1029/2007GL032921.

[16] A. Marshak, G. Wen, J. A. Coakley, L. A. Remer, N. G. Loeb, and R. F. Cahalan, "A simple model of the cloud adjacency effect and its impact on retrieved aerosol properties in the vicinity of clouds," J. Geophys. Res., vol. 113, p. D14 S17, 2008, DOI: 10.1029/2007JD009196.

[17] L. A. Remer, Y. J. Kaufman, D. Tanre, S. Mattoo, D. A. Chu, J. V. Martins, R. R. Li, C. Ichoku, R. C. Levy, R. G. Kleidman, T. F. Eck, E. Vermote, and B. N. Holben, "The MODIS aerosol algorithm, products, and validation," J. Atmos. Sci., vol. 62, no. 4, pp. 947-973, Apr. 2005.

[18] R. Levy, G. Leptoukh, R. Kahn, V. Zubko, A. Gopalan, and L. Remer, "A critical look at deriving monthly aerosol optical depth from satellite data," IEEE Trans. Geosci. Remote Sens., vol. 47, no. 8, pp. 2942-2956, Aug. 2009.

[19] B. Efron and G. Gong, "A leisurely look at the bootstrap, the jackknife, and cross-validation," Amer. Stat., vol. 37, no. 1, pp. 36-48, 1983. 